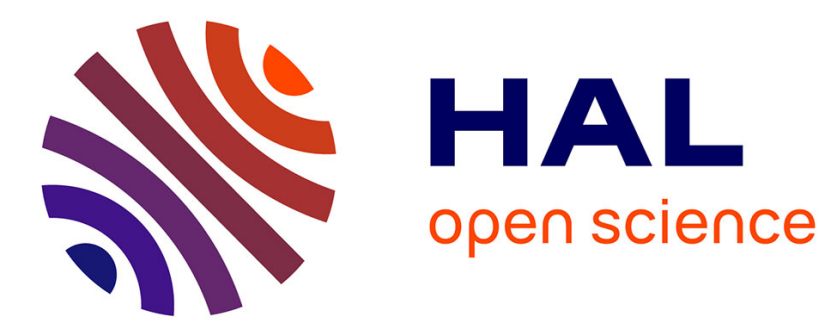

\title{
Depletion gels from dense soft colloids: Rheology and thermoreversible melting
}

Domenico Truzzolillo, D. Vlassopoulos, A. Munam, M. Gauthier

\section{To cite this version:}

Domenico Truzzolillo, D. Vlassopoulos, A. Munam, M. Gauthier. Depletion gels from dense soft colloids: Rheology and thermoreversible melting. Journal of Rheology, 2014, 58 (5), pp.1441-1462. 10.1122/1.4866592 . hal-01058961

\section{HAL Id: hal-01058961 https://hal.science/hal-01058961}

Submitted on 8 Jun 2021

HAL is a multi-disciplinary open access archive for the deposit and dissemination of scientific research documents, whether they are published or not. The documents may come from teaching and research institutions in France or abroad, or from public or private research centers.
L'archive ouverte pluridisciplinaire HAL, est destinée au dépôt et à la diffusion de documents scientifiques de niveau recherche, publiés ou non, émanant des établissements d'enseignement et de recherche français ou étrangers, des laboratoires publics ou privés. 


\title{
Depletion gels from dense soft colloids: Rheology and thermoreversible melting
}

\author{
Domenico Truzzolillo and Dimitris Vlassopoulos ${ }^{\text {a) }}$ \\ FORTH, Institute of Electronic Structure and Laser, and Department of Materials \\ Science and Technology, University of Crete, 70013 Heraklion, Crete, Greece
}

\author{
Abdul Munam and Mario Gauthier \\ Department of Chemistry, Institute for Polymer Research, University of Waterloo, \\ Waterloo, Ontario N2L 3G1, Canada
}

(Received 20 December 2013; final revision received 2 February 2014; published 27 August 2014)

\begin{abstract}
Synopsis
Upon addition of small nonadsorbing linear polymers, colloidal glasses composed of large hard spheres melt and eventually revitrify into the so-called attractive glass regime. We show that, when replacing the hard spheres by star polymers representing model soft particles, a reentrant gel is formed. This is the result of compression and depletion of the stars due to the action of the osmotic pressure from the linear homopolymers. The viscoelastic properties of the soft dense gel were studied with emphasis on the shear-induced yielding process, which involved localized breaking of elements with a size of the order of the correlation length. Based on these results, a phenomenological attempt was made at describing the universal rheological features of colloid/nonadsorbing polymer mixtures of varying softness. The star gel was found to undergo thermoreversible melting, despite the fact that conventional hard-sphere depletion gels are invariant to heating. This phenomenon is attributed to the hybrid internal microstructure of the stars, akin to a dry-to-wet brush transition, and is characterized by slow kinetics, on the time scale of the osmotic gel formation process. These results may be useful in finding generic features in colloidal gelation, as well as in the molecular design of new soft composite materials. (C) 2014 The Society of Rheology. [http://dx.doi.org/10.1122/1.4866592]
\end{abstract}

\section{INTRODUCTION}

Suspensions of particles interacting via a potential including an attractive component and which are space filling, also known as colloidal gels, have been a topic of significant scientific activity for many years because they combine great scientific and technological challenges [Mewis and Wagner (2012); Russel et al. (1989); Mezzenga and Fischer (2013); Zaccarelli (2007); Trappe and Sandkühler (2004)]. Indeed, altering the strength and range of the attractions at the origin of colloidal gelation enables tailoring the properties of colloidal dispersions to wider-ranging and challenging applications, from foodstuff and cosmetics to pharmaceutical and chemical products. In the recent years, advances in modeling and simulation [Zaccone et al. (2009); Zaccarelli (2007); Del Gado and Kob (2007);

\footnotetext{
${ }^{\text {a)} A u t h o r ~ t o ~ w h o m ~ c o r r e s p o n d e n c e ~ s h o u l d ~ b e ~ a d d r e s s e d ; ~ e l e c t r o n i c ~ m a i l: ~ d v l a s s o @ i e s l . f o r t h . g r ~}$
} 
Puertas et al. (2004); Cates et al. (2004); Ramakrishan et al. (2002); Mao et al. (1995)] and the emergence of (hard) colloid-polymer mixtures as an ideal model system for exploring the whole range of colloidal behaviors from dilute gels to dense glasses [Pham et al. (2002); Poon (2002)] gave a new boost to the field and triggered new efforts (experimental and modeling/simulations) toward the fundamental understanding of phenomena related to colloidal gelation. Examples of this include their mechanics and yielding, aging (coarsening), the relation between gelation and phase separation, linking to biological systems (e.g., proteins) or to nanocomposites, and the role of particle shape and softness. This work attempts to contribute to the latter topic, i.e., to investigate some rheological properties of gels made of soft colloidal particles. Hence, as this is not a review article, we shall directly focus on mixtures of colloids and nonadsorbing homopolymers.

Mixtures of hard sphere colloids and nonadsorbing polymers have been used extensively as interesting model systems to induce gelation via the depletion mechanism and their properties extensively studied [Asakura and Oosawa (1958); Vrij (1976); Vincent et al. (1986); Tuinier et al. (2000); Poon (2002); Shah et al. (2003); Lekkerkerker and Tuinier (2011)]. However, the seminal work of Pham et al. (2002) demonstrated that with this system, one can span the whole colloidal morphology diagram simply via entropic interactions. More importantly, they discovered a new state in the high particle volume fraction regime, the attractive glass, which is due to the depletion effect of linear chains on the particles. In particular, at high colloid volume fractions (about 0.6) in the (repulsive) glass state, adding linear polymer (having dimensions corresponding to about $10 \%$ of the size of the colloid so as to ensure short range depletion attraction) results in initial melting of the glass. However, enhancing the depletion strength by adding more polymer leads to the reentrant state which has been identified with an attractive glass, since in addition to the topological constraints of the dense colloids (cage effect), there is particle-particle bonding [Pham et al. (2002); Pham et al. (2008)]. Therefore, upon addition of small polymer chains, a repulsive colloidal glass made of large hard spheres will melt and eventually revitrify into an attractive glassy state. From the rheological standpoint, dynamic strain sweep experiments indicated that whereas a repulsive glass is characterized by one yield point (yield stress/yield strain) signaling the breaking of the cage, an attractive glass exhibits two yield points which refer to the two length scales in that system, associated with particle-particle bonds and particle cages [Pham et al. (2008)]. At the time, the so-called double yielding behavior was proposed as a signature of attractive glasses and was later confirmed in other studies involving glasses from both spherical and nonspherical particles [Kramb and Zukoski (2011); Koumakis and Petekidis (2011)]. However, double yielding is not a signature unique to attractive glasses. It can also characterize shear-induced melting of colloidal gels made from the same colloidpolymer mixtures at lower colloid volume fractions, due to the different length scales involved [Koumakis and Petekidis (2011)]. In fact, shear yielding in a variety of systems from emulsions to foams and nanocomposites, which often consist of gel-like networks, is a macroscopic process that takes place via a gradual evolution involving microscopic structural rearrangements [Rouyer et al. (2003); Osman et al. (2004); Masschaele and Vermant (2009); Putz and Burghelea (2009); Hsiao et al. (2012); Kamble et al. (2013); Moll et al. (2013)].

The colloid-polymer paradigm has also been extended to soft particles. In particular, softness of the interactions and the particles has been shown to dramatically alter nonergodic-to-ergodic transitions leading to multiple glassy states [Stiakakis et al. (2002); Stiakakis et al. (2005); Eckert and Bartsch (2002, 2003); Datta et al. (2011); Truzzolillo et al. (2011, 2013a); Wiemann et al. (2012)]. In our work, we employed multiarm star polymers as model soft colloids encompassing the two main macroscopic response 
features of entropically interacting soft particles, namely, squeezing and interpenetration [Bonnecaze and Cloitre (2010); Vlassopoulos and Fytas (2010)]. Moreover, these systems are well-characterized, with a pair-interaction potential which is tunable at the level of the molecules (by varying length and number of arms) and so are their macroscopic properties [Likos et al. (1998a); Vlassopoulos et al. (2001)]. Earlier studies confirmed that depletion is at work in these soft colloid-homopolymer mixtures, and indeed repulsive star glasses melt upon addition of smaller polymer chains, though the range of size ratios over which this effect is observed appears broader as compared to the hard sphere case. At the same time, it was shown that the osmotic pressure due to the linear polymers is imparted on the stars through both depletion (like in hard spheres) and compression effects [Stiakakis et al. (2002); Stiakakis et al. (2005); Truzzolillo et al. (2011)].

We now extend that work and address the effect of enhanced osmotic pressure due to a higher concentration of added linear polymer chains. We show that due to substantial star compression, their effective volume fraction is drastically reduced and hence the observed reentrant state is a gel and not an attractive glass. The rheological properties corroborate this assignment. An attempt is also made at comparing hard and soft colloid-polymer mixtures, in order to phenomenologically define the universality of their behavior and identify key differences. Further, we show the importance of the internal microstructure of the particles, which appears responsible for the recently reported thermal melting of these entropic gels [Truzzolillo et al. (2013b)]. We show that this phenomenon is thermoreversible and characterized by kinetics identical to an aging rejuvenated gel. Hence, soft colloidal gels are tunable and can form a plethora of morphologies with intriguing properties.

The manuscript is organized as follows: The experimental details are discussed in Sec. II. Then, Sec. III presents and discusses the experimental results on the formation and properties of the reentrant star gel and its thermoreversible melting. Section IV compares qualitatively the general rheological properties of depletion gels prepared from soft and hard colloids. Finally, Sec. V summarizes the main findings from this work.

\section{EXPERIMENTAL SYSTEMS AND METHODS}

\section{A. Materials}

A multiarm polybutadiene star(s) sample (PBD, with more than $90 \%$ of 1,4-butadiene units), coded as s362-2, was employed as model soft spheres. The star had a numberaverage branching functionality $f=362$ arms (and a weight-average branching functionality of 392), a total weight-average molar mass $M_{\mathrm{w}}{ }^{\mathrm{s}}=9.8 \times 10^{6} \mathrm{~g} / \mathrm{mol}$, and polydispersity index PDI $=M_{\mathrm{w}} / M_{\mathrm{n}}=1.14$, whereas each arm had $M_{\mathrm{w}}{ }^{\mathrm{a}}=24400 \mathrm{~g} / \mathrm{mol}$ and PDI $=1.06$ [Gauthier and Munam (2010)]. A trace from size exclusion chromatography (SEC) analysis of the stars after fractionation, based on the signal of the light scattering detector, is depicted in Fig. 1(A). This chromatogram is fitted reasonably well with a Lorentz-type function. In fact, the inability to improve the fitting by employing more than one Lorentz-type peak functions is consistent with the virtual absence of contaminants in that sample. According to the Daoud-Cotton model (1982), a star polymer in a good solvent is characterized by a nonhomogeneous monomer density distribution consisting of three regions: The inner meltlike core, the intermediate ideal region, and the outer excluded volume region, where interactions with neighboring stars may take place [see Fig. 1(B)]. These systems are in fact hybrids since the (carbosilane dendrimer) core, on which the arms are grafted, has a chemically different composition, albeit it is also small. In fact, we calculated a radius for the carbosilane $\left(\mathrm{C}_{308} \mathrm{H}_{612} \mathrm{Si}_{61}\right)$ dendritic core $R_{\text {core }}=3.17 \mathrm{~nm}$ by taking into account the $\mathrm{C}-\mathrm{C}$ bond length $(0.154 \mathrm{~nm})$, the $\mathrm{Si}-\mathrm{C}$ bond length $(0.185 \mathrm{~nm})$, and 

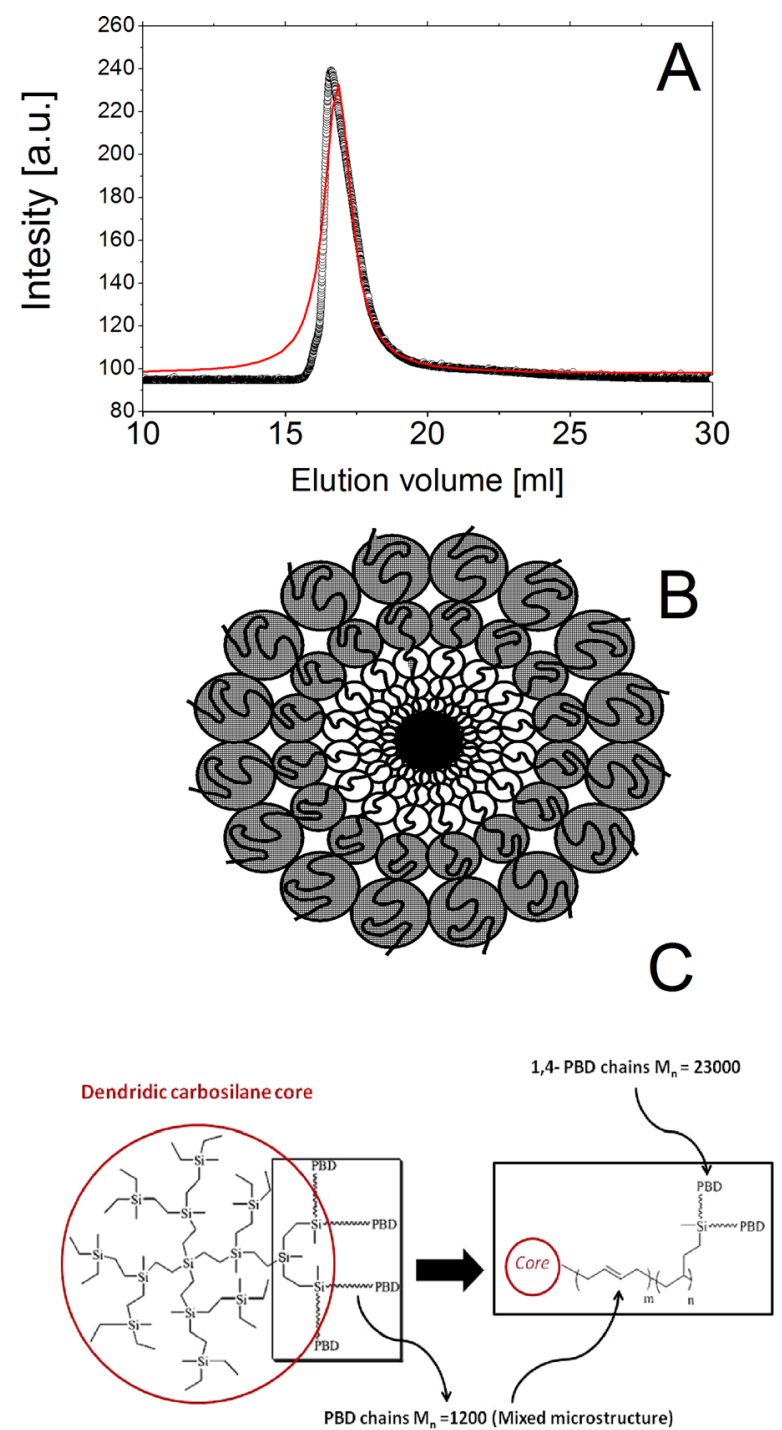

FIG. 1. (A) Light scattering signal in SEC analysis of the star polymer (symbols). The curve represents a fit performed employing a single Lorentz-type function. (B) Two-dimensional representation of a star polymer (here with 16 arms) in a good solvent according to the Daoud-Cotton model (see text). According to the model, each arm consists of spherical blobs (i.e., regions of space uncrossed by other arms), whose size increases from the center to the outer region of the star. The monomer density varies accordingly from the center outward: From the inner meltlike core (black) to the intermediate ideal-chain region and to the outer swollen region (shaded). Only the swollen blobs at the periphery can interact (i.e., interpenetrate) with neighboring stars or chains. (C) Dendritic carbosilane core structure after coupling the Si-sites with mixed microstructure polybutadiene arms having $M_{\mathrm{n}} \approx 1200 \mathrm{~g} / \mathrm{mol}$. Here, $m=0.48$ and $n=0.52$, where $m$ and $n$ are the mole fractions of 1,4-butadiene and 1,2-butadiene structural units, respectively. Right: Overall structure of the hybrid star polymer after coupling the hydrosilylated starlike polybutadiene with 1,4-polybutadiene chains $\left(M_{\mathrm{n}}=23000 \mathrm{~g} / \mathrm{mol}\right)$.

the Si-C-C bond angle (109.5 ) [Zhou and Roovers (1993); Roovers and Ding (2009)]. Figure $1(\mathrm{C})$ illustrates the molecular details of the carbosilane dendritic core and the coupling of linear polybutadiene chains to form the star [Gauthier and Munam (2010)]. The linear 1,4-polybutadiene chains (L) used, coded as c6, were obtained from Polymer Source (Canada) and had a weight-average molar mass $M_{\mathrm{w}}{ }^{\mathrm{L}}=6000 \mathrm{~g} / \mathrm{mol}$. 
The polymers were dissolved in squalene, a nearly athermal and nonvolatile solvent. The hydrodynamic radii were determined from dynamic light scattering (DLS) measurements in dilute solution at $20^{\circ} \mathrm{C}$ and found to be $R_{\mathrm{h}}{ }^{\mathrm{s}}=40 \mathrm{~nm}$ and $R_{\mathrm{h}}{ }^{\mathrm{L}}=2 \mathrm{~nm}$. Their respective overlap concentrations were $c_{\mathrm{s}}^{*}=60.6 \mathrm{mg} / \mathrm{ml}$ and $c_{\mathrm{L}}{ }^{*}=297 \mathrm{mg} / \mathrm{ml}$. A star polymer solution without added linear chains was prepared at an apparent volume fraction (based on single star size) $\Phi_{\mathrm{s}}=c_{\mathrm{s}} / c_{\mathrm{s}} *=3.75$, corresponding to the glassy state. When preparing mixtures with linear chains, the same fraction (number density) of star polymers was maintained in the samples. This means practically that the added linear chains replaced the squalene solvent, and hence, at high enough fractions of chains, there was no squalene left and the mixture became binary.

\section{B. Rheology}

To investigate the state of the star/linear mixtures, we used rheological measurements, which were carried out with a sensitive strain-controlled rheometer (ARES-HR 100FRTN1 from TA USA, formerly Rheometric Scientific). Due to the very limited amount of samples available, a home-made cone-and-plate geometry (stainless steel cone of $8 \mathrm{~mm}$ diameter and $0.166 \mathrm{rad}$ cone angle) was used. The temperature was set at $20.00 \pm 0.01{ }^{\circ} \mathrm{C}$ and controlled by a Peltier plate with a recirculating water/ethylene glycol bath. During an experimental run, the sample (which was solidlike in appearance) was loaded on the rheometer, and a well-defined preshear protocol was applied in the following sequence: The sample was subjected to (i) a dynamic strain amplitude sweep at a fixed frequency $(1 \mathrm{rad} / \mathrm{s})$, to determine the range of strains corresponding to liquidlike response; (ii) a dynamic time sweep at a large nonlinear strain selected within this range (200\%) and at the same frequency, to shear-melt and hence fully rejuvenate the sample, as judged by the time-independent apparent moduli (this step lasted typically $300 \mathrm{~s}$ ); (iii) after a set rest time, i.e., a waiting time $t_{\mathrm{w}}$ (typically about $10^{5} \mathrm{~s}$ ), a dynamic time sweep was performed in the linear regime to follow the time evolution of the moduli to nearly constant values, corresponding to the aged sample; (iv) small-amplitude oscillatory shear (SAOS) tests were carried out within the frequency range $0.01-100 \mathrm{rad} / \mathrm{s}$; (v) large amplitude oscillatory shear (LAOS) was performed via dynamic strain sweep tests that were carried out at fixed frequencies ( 1 and $10 \mathrm{rad} / \mathrm{s})$, with strain amplitudes covering the range from the linear $\left(\gamma_{0} \leq 1 \%\right)$ to the strongly nonlinear $\left(\gamma_{0} \approx 250 \%\right)$ regimes; (vi) the reentrant solid (gel, see Sec. III) was further examined in start-up shear experiments at several shear rates in the range $0.001 \mathrm{~s}^{-1} \leq \dot{\gamma} \leq 0.06 \mathrm{~s}^{-1}$; and finally (vii) SAOS tests were also performed on the reentrant solid at different temperatures within the range 20-90 ${ }^{\circ} \mathrm{C}$. To heat the sample from one temperature to the next where the SAOS measurements were performed, a temperature ramp test was carried out at $0.01 \mathrm{rad} / \mathrm{s}$ and a rate of $2.5^{\circ} \mathrm{C} / \mathrm{min}$.

\section{RESULTS AND DISCUSSION}

\section{A. Linear viscoelasticity, aging, and morphology of the mixtures}

Figure 2 depicts the results from the SAOS measurements with the mixtures at different fractions of linear chains. One can observe both solidlike and liquidlike behaviors. In the absence of linear chains $\left(\Phi_{\mathrm{L}}=0\right)$, the concentrated star solution with $\Phi_{\mathrm{s}}=3.75$ exhibits features typical of glassy behavior: Both the storage $\left(G^{\prime}\right)$ and loss $\left(G^{\prime \prime}\right)$ moduli are only weakly frequency-dependent with $G^{\prime \prime}$ reaching a minimum, and $G^{\prime}(\omega)>G^{\prime \prime}(\omega)$ over four decades of frequency [Fig. 2(A)]. Note that solidlike behavior (glass here and gel below) is associated with strong aging (see Fig. 4). Upon addition of linear chains at 


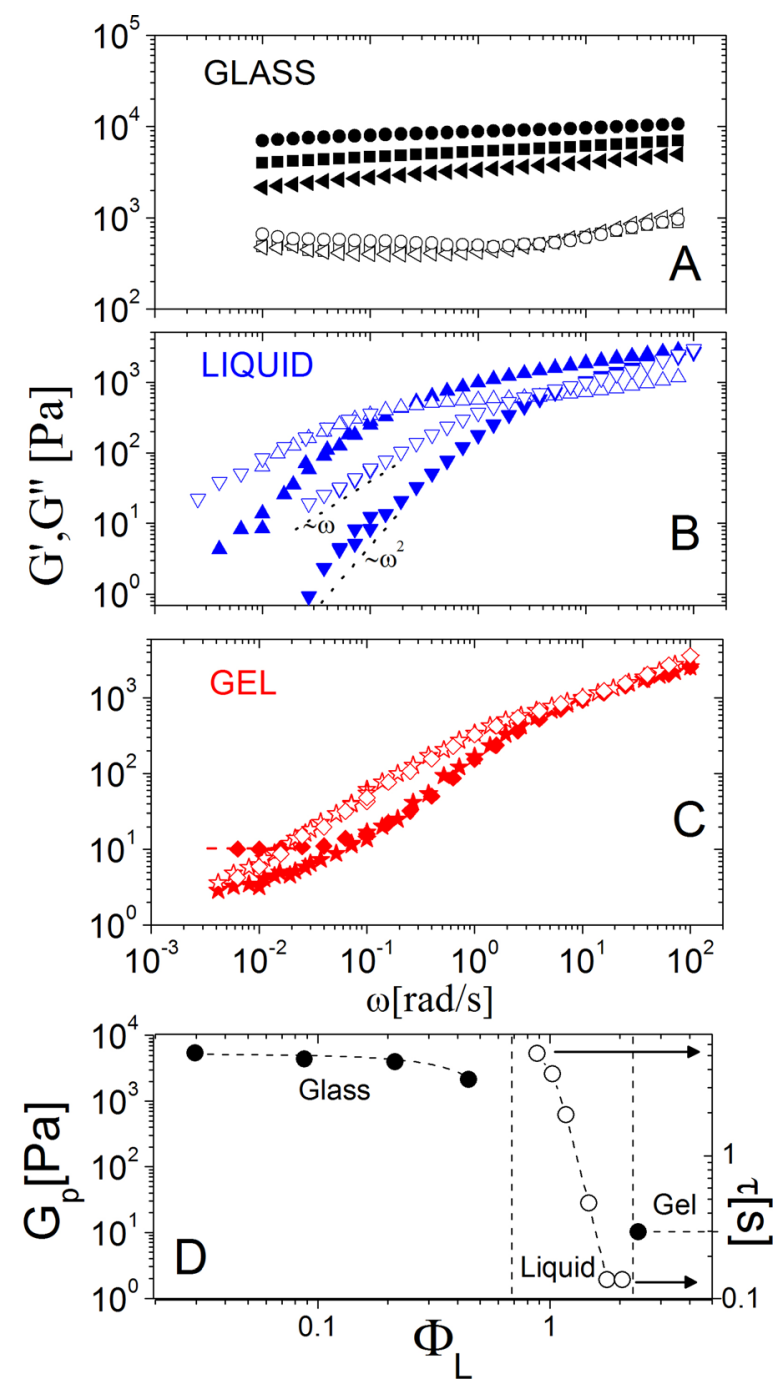

FIG. 2. Linear viscoelastic properties for $\mathrm{s} 362-2 / \mathrm{c} 6$ mixtures with $\Phi_{\mathrm{s}}=3.75$ and different volume fractions of linear chains. In all the panels: Full symbols $\rightarrow G_{\mathrm{p}}$ or $G$, empty symbols $\rightarrow \tau$ or $G$. (A) (Glass, modulus): $\Phi_{\mathrm{L}}=0$ (circles), $\Phi_{\mathrm{L}}=0.21$ (squares), $\Phi_{\mathrm{L}}=0.43$ (left triangles). (B) (Liquid, relaxation time): $\Phi_{\mathrm{L}}=0.88$ (up triangles), $\Phi_{\mathrm{L}}=1.78$ (down triangles). (C) (Gel, modulus): $\Phi_{\mathrm{L}}=2.18$ (stars), $\Phi_{\mathrm{L}}=2.24$ (lozenges). (D) The plateau moduli of the arrested states (closed circles, left vertical axis) and the relaxation times of the ergodic phases (open circles, right vertical axis) observed for a star polymer concentration $\Phi_{\mathrm{s}}=3.75$ as a function of the linear polymer concentration $\Phi_{\mathrm{L}}$. The vertical lines indicate the solid-liquid and liquid-solid transitions. Lines through the symbols are drawn to guide the eye. The horizontal arrows in the liquid region (B) point to the relaxation time axis.

increasing $\Phi_{\mathrm{L}}$ while maintaining the star fraction fixed at $\Phi_{\mathrm{s}}=3.75$, the moduli become increasingly sensitive to frequency variations [Fig. 2(A)]. In fact, the added polymers induce remarkable softening of the initial glassy suspensions, and eventually a transition to the liquid state $\left(G^{\prime} \sim \omega^{2}<G^{\prime \prime} \sim \omega^{1}\right)$ above a certain apparent volume fraction of the linear polymer [Fig. 2(B)]. Upon further addition of linear chains (for a fraction $\Phi_{\mathrm{L}}>2.18$ ), the mixture undergoes a reentrance transition to another solidlike state, as shown by the low frequency crossover with $G^{\prime} \sim \omega^{0}>G^{\prime \prime}\left[\omega_{\mathrm{c}} \sim 10^{-2} \mathrm{rad} / \mathrm{s}\right.$, see Fig. 2(C)]. This state is characterized by a low plateau modulus $\left(G_{\mathrm{p}}{ }^{\mathrm{r}} \approx 10 \mathrm{~Pa}\right)$, three 
orders of magnitude lower than the initial glassy phase $\left(G_{\mathrm{p}}{ }^{\mathrm{g}} \approx 10^{4} \mathrm{~Pa}\right)$. A compilation of the data obtained at the different regimes is depicted in Fig. 2(D) in the form of plateau modulus (of the solid) or relaxation time (of the liquid) as function of added linear polymer concentration. One can appreciate the osmotic effects of the linear polymer in decreasing the modulus of the glass. The peculiar dependence of relaxation time on the linear polymer concentration can be rationalized by the fact that the liquid region is constrained by (different) solids on both sides, hence the relaxation time (or viscosity) increases as either of the two limits is approached (the relaxation time actually diverges at the solid transition). The minimum in the curve (almost at the border of the liquid-solid transition here) should mark the onset of dominance of depletion-induced attraction in the mixture. Note that the maximum calculated star shrinkage (discussed below) occurs when all the solvent is replaced by linear polymer in the gel region. The qualitative difference between the reentrant solid (at $\Phi_{\mathrm{L}}=2.24$ ) and the original repulsive glass can be clearly observed in Fig. 3. Note that for $\Phi_{\mathrm{L}}=2.24$, all the squalene solvent is replaced by linear chains. However, this is just a peculiarity of the specific combination of colloid fraction and size ratio used in the experiment, and it does not have any effect on the properties of the systems discussed here.

The very low value of $G_{\mathrm{p}}{ }^{\mathrm{r}}$ implies that the reentrant state is not an attractive glass, in contrast to previous experiments and simulations with hard-colloid/polymer mixtures [Pham et al. (2008)]. Note that the key difference between the two states (repulsive glass and reentrant solid) is the presence or not of caging [Truzzolillo et al. (2011)]. Furthermore, attractive glasses (exhibiting both caging and attractions) are characterized by a larger storage modulus when compared to the corresponding repulsive glasses (with the same number density of particles) [Pham et al. (2008); Koumakis and Petekidis (2011)]. We, therefore, argue that the star-linear polymer mixture exhibits a unique repulsive glass to liquid to reentrant gel transition upon increasing the linear polymer concentration. The formation of a gel can be explained by taking into account the osmotic compression of the star polymers in the presence of linear chains. This is based on a recent self-consistent mean field approach to evaluate the size of star polymers in linear

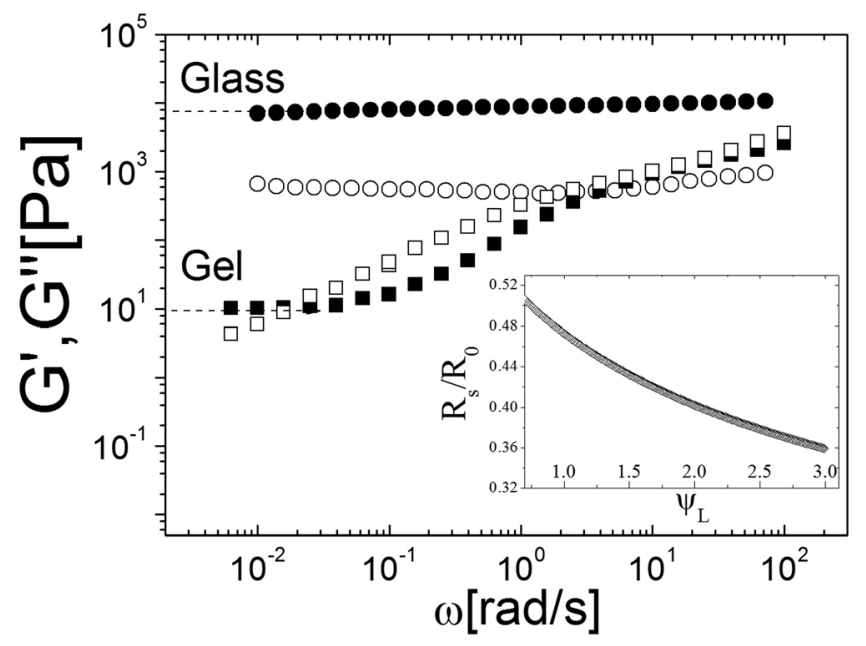

FIG. 3. Linear viscoelastic spectra for mixtures $s 362-2 / \mathrm{c} 6$ with $\Phi_{\mathrm{s}}=3.75$ and linear polymer fractions $\Phi_{\mathrm{L}}=0$ (bold symbols) and $\Phi_{\mathrm{L}}=2.24$ (open symbols). Inset: Predicted osmotic shrinkage of the star, depicted as the star radius in the mixture (normalized to that of a single star in squalene) as a function of the fraction of added polymer (see text). 
polymer solutions or melts [Truzzolillo et al. (2011)]. At equilibrium, the size of a single star polymer in a linear polymer chains solution, with a degree of polymerization $N_{\mathrm{c}}$, can be calculated by solving the following equation for the equilibrium of forces on the star [Wilk et al. (2010)]:

$$
\frac{3 f a R_{\mathrm{s}}}{N_{\mathrm{s}}}+4 \pi b^{3} R_{\mathrm{s}}{ }^{2} \delta^{-3} N_{\mathrm{s}}{ }^{-3 \nu} f^{-\frac{3}{5}} \psi_{L}\left[1+P\left(\psi_{L}\right)\right]-\frac{3 v N_{\mathrm{s}}{ }^{2} f^{2} a^{3}}{2 R_{\mathrm{s}}{ }^{4}}=0 .
$$

Here, $a$ is the monomer statistical length, $\delta$ is the chain/star size ratio, $N_{\mathrm{s}}$ is the degree of polymerization of the star arms, $\psi_{\mathrm{L}}$ is the effective volume fraction of chains in the mixture excluding the stars (different from the fraction of linear chains in the mixture including stars, $\left.\Phi_{\mathrm{L}}\right), v=1$ is the excluded volume parameter under good solvency conditions, and $\nu=3 / 5$ is the Flory exponent. $P\left(\psi_{\mathrm{L}}\right)=\left[\left[c_{\mathrm{L}} \Pi\left(\psi_{\mathrm{L}}\right)\right] / k_{\mathrm{b}} T\right]-1$ is a function of the osmotic pressure $\Pi\left(\psi_{\mathrm{L}}\right)$ exerted by the chains on the star and can be computed as [Wilk et al. (2010)]

$$
P(x)=\frac{1}{2} x \exp \left\{\frac{1}{4}\left[\frac{1}{x}+\left(1-\frac{1}{x}\right) \ln (x+1)\right]\right\} \text { with } x\left(\psi_{\mathrm{L}}\right)=\frac{1}{2} \psi_{\mathrm{L}} \pi^{2}\left[1+\frac{1}{4}\left(\ln 2+\frac{1}{2}\right)\right] .
$$

For a given amount of linear chains added to the star polymer solution, we solved recursively Eq. (1) until it converged to stable values $\left(\psi_{\mathrm{L}}, R_{\mathrm{S}}\right)$ of linear-chain volume fraction and star-polymer dimension that univocally defined the mixtures. This procedure is conceptually valid if the monomer-solvent interaction parameter $v$ can be considered close to one. This calculation allows the estimation of the normalized radius of the star polymers in the mixture with $76 \mathrm{wt} . \%\left(\psi_{\mathrm{L}}=3.0\right.$ or equivalently $\left.\Phi_{\mathrm{L}}=2.24\right)$ of linear chains in the absence of molecular solvent. This calculation yielded the normalized radius of the star polymers in a mixture with linear chains at $\psi_{\mathrm{L}}=3.0$. The normalized radius $R_{\mathrm{S}} / R_{0}$ thus found was 0.36 , corresponding to an apparent star volume fraction $\Phi_{\mathrm{S}}=0.17$ (significantly reduced as compared to the original fraction $\Phi_{\mathrm{S}}=3.75$ ). Note that this value of the radius is in good agreement with the value estimated by the Daoud-Cotton approach extended to high molecular weight solvents [Raphael et al. (1993)] $R_{\mathrm{S}} / R_{0}=N_{\mathrm{L}}^{-1 / 5}=0.39$. The inset of Fig. 3 depicts the calculated dependence of the calculated $R_{\mathrm{S}} / R_{0}$ on $\psi_{\mathrm{L}}$. Hence, the star volume fraction is drastically reduced from $\Phi_{\mathrm{s}}=3.75$ to $\Phi_{\mathrm{s}}=0.17$ as a result of the osmotic pressure of the linear chains. This is a unique feature of these soft spheres and the value of $\Phi_{\mathrm{s}}$, along with the respective modulus $G_{\mathrm{p}}$ r , conform to the assignment of the reentrant solid to gel. Although we do not have direct structural information about the particular gel (due to the very limited amount of sample), we do expect from the literature that at this volume fraction, the shrunk stars form a percolated network [Rueb and Zukoski (1997); Varadan and Solomon (2001); Cates et al. (2004)]. This is further discussed below.

Therefore, the addition of linear chains alters the macroscopic behavior of the starlinear polymer mixtures via a star size reduction due to the osmotic pressure exerted by the chains, as quantified above. This effect can be regarded as entropic dewetting of the effective star solvent (squalene and/or linear chains) whose quality decreases [Raphael et al. (1993); Reiter et al. (2000); Matsen et al. (2001)]. Dewetting is closely associated with an accompanying depletion mechanism that introduces an attractive component in the pair interaction potential between stars [Stiakakis et al. (2005)]. Since the linear chains must pay a huge entropic cost to fully wet the soft particles, they are preferentially depleted from the contact region between two multiarm stars, which produces a net attraction between them. Note that, whereas normally depletion forces are at work at low 
linear polymer concentrations [Camargo and Likos (2010); Stiakakis et al. (2005)], they are also responsible for the dewetting of the chains from the stars. In fact, they can be also seen as arising from the decreased quality of the effective solvent (linear chains and squalene) for entropic reasons. It is also worth mentioning that even in the absence of molecular solvent (as is the in the case here for $\Phi_{\mathrm{L}}=2.24$ and higher), the concept of depletion was invoked to explain brush collapse in the case of end-grafted polymer brushes in a polymer matrix [Hoy and Grest (2007)]. Hence, softness is responsible for the novel reentrant gel state originating from a repulsive glass in this model soft sphere mixture.

Aging is important and unavoidable when dealing with metastable states. Here, we have accounted for aging, but its mechanism and consequences on the properties of star glasses and gels are beyond the scope of the present work. However, by considering aging, we ensured that the various morphologies discussed are all long-time aged morphologies or else quasisteady state. We used dynamic time sweep tests to investigate the time evolution of the moduli for the different star-linear polymer mixtures, starting from the repulsive glass at $\Phi_{\mathrm{S}}=3.75$ without added linear polymer $\left(\Phi_{\mathrm{L}}=0\right)$, up to the reentrant gel at the highest linear polymer volume fraction achievable $\left(\Phi_{\mathrm{L}}=2.24\right)$. We show representative results for the three different morphologies encountered. The repulsive glass [Fig. 4(A)] is characterized by a slow logarithmic increase in $G^{\prime}$ and a decrease in $G^{\prime \prime}$, typical for this type of star glass [Erwin et al. (2011)] and other soft colloidal glasses [Ramos and Cipelletti (2005)]. The liquid phase at $\Phi_{\mathrm{L}}=1.78$ does not age, as expected [Fig. 4(B)]. Finally, the gel state at $\Phi_{\mathrm{L}}=2.24$ ages markedly but with kinetics very different from the glass: $G^{\prime}$ exhibits an S-shaped increase after a long time, whereas $G^{\prime \prime}$ is

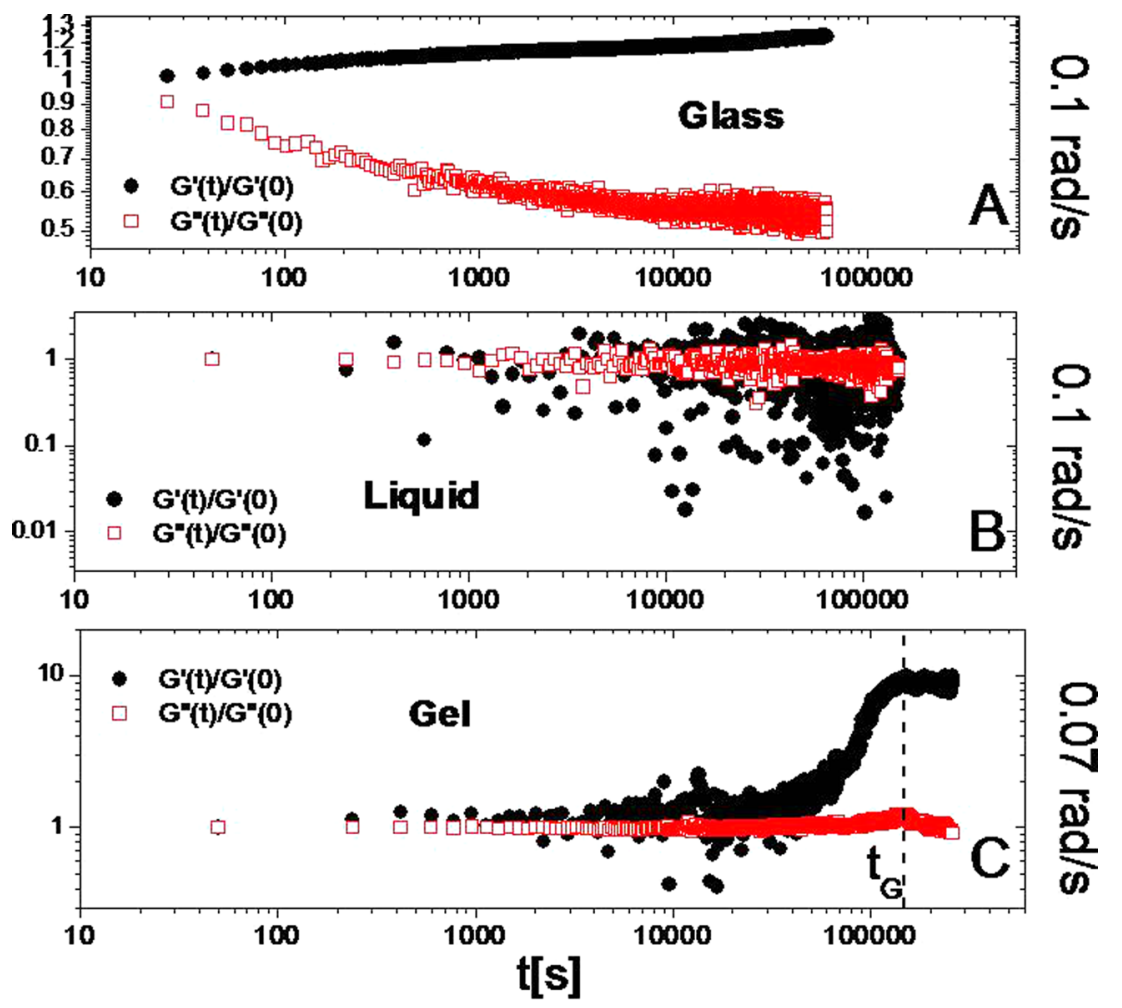

FIG. 4. Time evolution of the moduli normalized $G^{\prime}$ and $G^{\prime \prime}$ at fixed frequencies for the glass (A), liquid (B), and gel (C) states. The moduli are normalized to their respective values at $t=0$, when the aging measurement starts after rejuvenation (see text). The three plots are taken at different frequencies based on the dynamic data of Fig. 2 . 
practically time-independent [Fig. $4(\mathrm{C})]$. What is remarkable here is the very long time (on the order of $10^{5} \mathrm{~s}$ ) needed to reach quasisteady state behavior, which rendered the experimental effort tedious.

\section{B. Nonlinear response and yielding}

\section{LAOS}

Selected LAOS data were collected and analyzed using Lissajous-Bowditch (LB) stress-strain plots [Rogers et al. (2011)] to determine the sensitivity of the different morphologies to this type of nonlinear deformation. The data obtained from the dynamic strain sweep measurements for a repulsive glass $\left(\Phi_{\mathrm{L}}=0\right)$ and a reentrant depletion gel $\left(\Phi_{\mathrm{L}}=2.24\right)$ are depicted in Figs. 5(A) and 5(B), respectively. The former exhibits the typical features of a repulsive glass with a single peak in $G^{\prime \prime}$, occurring at the $G^{\prime}-G^{\prime \prime}$ crossover, which also marks the yield strain amplitude (about $25 \%$ in this case). On the other hand, the moduli crossover occurs at about $60 \%$ for the gel, and no $G^{\prime \prime}$ overshoot is observed. Note also that, at this frequency, the moduli of the gel are nearly identical and orders of magnitude lower than for the glass. The low $G_{\mathrm{p}}$ and large yield strain observed for the gel (in contrast to the glass) reflect its ductile-like breaking, which is expected for colloidal gels and networks [Shih et al. (1990); Moll et al. (2013); Rueb and Zukoski (1997)]. We also remark that the yielded glass reached a $G^{\prime}$-to- $G^{\prime \prime}$ slope (versus strain amplitude) ratio of about 2 , as seen often in solid-liquid transitions [Koumakis et al. (2012)], whereas this is not the case for the gel. This may be suggestive of the gradual nature of gel's yielding process, with localized bond breaking events taking place over a wide range of strains [Masschaele and Vermant (2009); Koumakis and Petekidis (2011); Moll et al. (2013); Hsiao et al. (2012)]. Furthermore, the repulsive glass exhibits a nonlinear stress-strain response characterized by a squarelike LB plot [Fig. 5(C)], where the
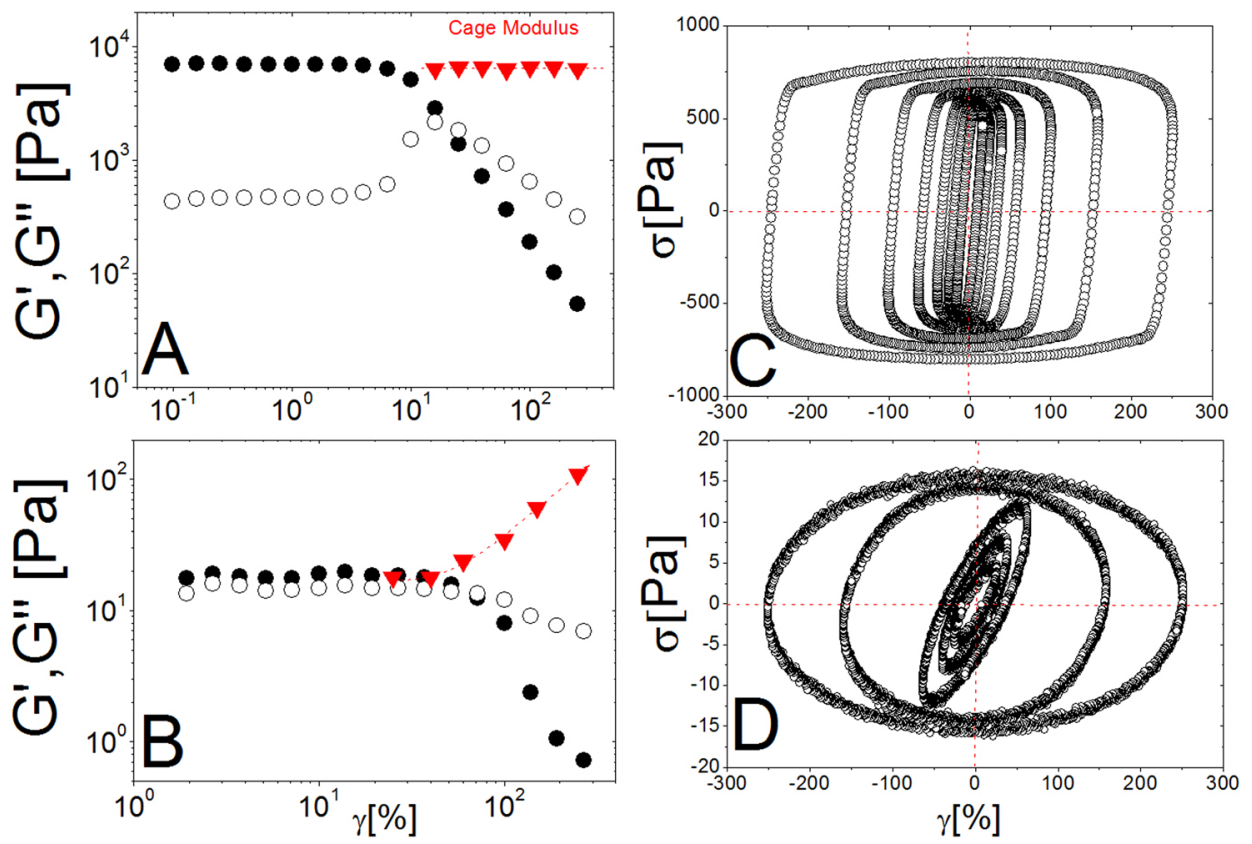

FIG. 5. Dynamic strain sweep and selected Lissajous plots for $[(\mathrm{A})$ and $(\mathrm{C})]$ mixture $\mathrm{s} 362-2 / \mathrm{c} 6\left(\Phi_{\mathrm{s}}=3.75\right.$, $\Phi_{\mathrm{L}}=0$; repulsive glass); [(B) and (D)] mixture s362-2/c6 $\left(\Phi_{\mathrm{s}}=3.75, \Phi_{\mathrm{L}}=2.24\right.$; gel). The cage modulus extracted from the Lissajous plots for large amplitude sweeps is plotted in panels A and B (red triangles). 
properties of the material are dictated by the yield of the star cages being able to reform within one deformation cycle. This is a feature typical of repulsive glasses, already observed in other colloidal glasses within the caging framework [Rogers et al. (2011); Truzzolillo et al. (2013a)]. The determined apparent cage modulus $G_{\text {cage }}=\left.(d \sigma / d \gamma)\right|_{\sigma=0}$ at different strain amplitudes is plotted in Fig. 5(A) along with the apparent $G^{\prime}$ and $G^{\prime \prime}$ from the dynamic strain sweeps. It is interesting to observe that whereas the first harmonic nonlinear moduli drop at large strain amplitudes beyond the yielding transition, due to the deformation and breaking of the cages, $G_{\text {cage }}$ remains equal to the low-strain linear $G^{\prime}$. During a cycle, the elastic response of such dense suspensions is dictated by the continuous formation of local microstructure whose response does not depend on the absolute amplitude of the oscillations. We argue that the insensitivity of $G_{\text {cage }}$ to strain amplitude reflects the dense state of the glass, i.e., caging.

The ergodic liquid phase resulting from the addition of linear chains is characterized by Newtonian flow and concentric, nearly symmetric LB ellipses (not shown) having their principal axes aligned with the Cartesian axes $(\sigma, \gamma)$ for $\omega=10^{-2} \mathrm{rad} / \mathrm{s}$ corresponding to a phase angle $\delta=\pi / 2$ between the stress and the strain, or equivalently $G^{\prime \prime} \gg G^{\prime}$. The reentrant gel is characterized by LB ellipses [Fig. 5(D)] with different strain amplitude-dependent orientations of the principal axes with respect to the $\sigma-\gamma$ axes, which signify the absence of caging [compared to Fig. 5(C)]. Beyond the yield point of the material [yield strain $\gamma_{\mathrm{y}} \approx 60 \%$-see Fig. 5(B)], the ellipses open up and become symmetrical [Fig. 5(D)]. Therefore, this situation is very different from the original repulsive glass, and its features conform to those of a weak gel, where the dynamical arrest is driven by attraction phenomena rather than by steric repulsive constraints. Upon shearing, the weak bonds between the stars break, hence the material flows $\left(\gamma_{0}>200 \%\right)$. Furthermore, in reference to the LB curves of Fig. 5(D), an apparent modulus is similarly extracted at zero stress, $G_{\text {app,gel }}=\left.(d \sigma / d \gamma)\right|_{\sigma=0}$. We distinguish it from the cage modulus because here the mixture of shrunk stars in a sea of linear polymers (and sometimes solvent as well) is not dense, as expected for a colloidal gel (see also cartoon of Fig. 8). At low strains $\left(\gamma<\gamma_{\mathrm{y}}\right), G_{\text {app,gel }}$ is identical to the linear viscoelastic modulus $G^{\prime}$. However, beyond yielding of the gel, it keeps on increasing with strain, whereas the first harmonic apparent moduli keeps dropping [Fig. 5(B)]. This is a signature for gel fluidization and reflects viscous resistence to flow. In fact, for a purely viscous liquid $\left.(d \sigma / d \gamma)\right|_{\sigma=0}$ becomes infinite.

\section{Start-up in simple shear}

To further characterize the depletion gel (at $20^{\circ} \mathrm{C}$ and $\Phi_{\mathrm{L}}=2.24$ ), we elucidated the yielding mechanism during start-up shear for different imposed shear rates within the range $0.001 \leq \dot{\gamma} \leq 0.06$, which encompasses the region corresponding to the appearance of $G_{\mathrm{p}}{ }^{\mathrm{r}}$ in the linear response. Figure 6(A) depicts the dependence of the measured stress on the accumulated strain for different values of imposed shear rate. The appearance of stress overshoots at low rates signals the (yielding) response of the depletion gel state: For the two highest shear rates, the stress increases with strain and gradually reaches a steady state. This response is typical of a liquidlike system where dissipative processes dominate the response, which here corresponds to the yielded gel. Note that these rates also correspond to the frequency regime where the system is liquidlike in Fig. 3, but invoking a Cox-Merz type of argumentation for such complex systems is not straightforward [Erwin et al. (2010)]; here, we only report this observation. At lower values of shear rates (below $0.035 \mathrm{~s}^{-1}$ ), after an initial linear increase with strain (or time), the stress reaches a maximum value that is commonly identified with the static yield stress $\sigma_{\mathrm{y}}$ and 

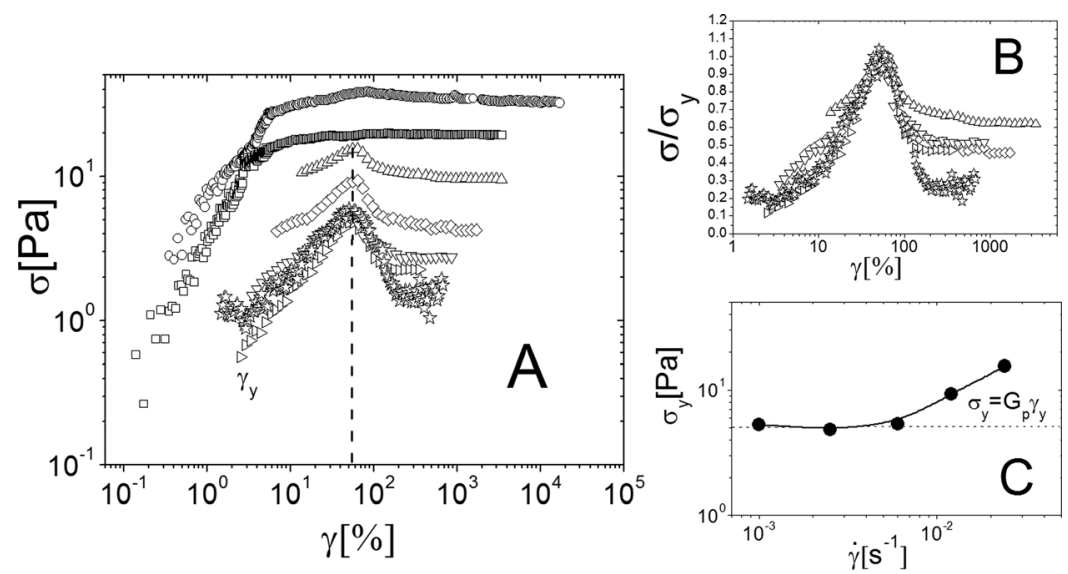

FIG. 6. (A) Stress $(\sigma)$ as a function of strain $(\gamma)$, i.e., the product of shear rate and time, during step rate experiments for the gel-like mixture s362-2/c6 with $\Phi_{\mathrm{L}}=2.24$ (no small molecule solvent) at different shear rates $\dot{\gamma}=0.06 \mathrm{~s}^{-1}$ (circles), $\dot{\gamma}=0.035 \mathrm{~s}^{-1}$ (squares), $\dot{\gamma}=0.024 \mathrm{~s}^{-1}$ (up-triangles), $\dot{\gamma}=0.012 \mathrm{~s}^{-1}$ (lozenges), $\dot{\gamma}=0.006 \mathrm{~s}^{-1}$ (reverse triangles), $\dot{\gamma}=0.0025 \mathrm{~s}^{-1}$ (right triangles), $\dot{\gamma}=0.001 \mathrm{~s}^{-1}$ (stars). The dashed line indicates the static yield point. (B) The data of (A) plotted as normalized stress (to the peak value, i.e., the yield stress) versus strain. The two highest rates from (A) correspond to the post-yield region (see also the respective frequencies in Fig. 3), i.e., not exhibiting a peak, and hence, these are not included in the plot. (C) The yield stress as a function of shear rate. The solid line is the best fit of the Eyring-type equation (see text). The horizontal dashed line indicates the yield stress plateau.

then decays to a steady-state value. Further, below a critical threshold $\dot{\gamma}<\dot{\gamma}_{\mathrm{c}} \sim 10^{-2} \mathrm{~s}^{-1}$, the peak stress appears to be independent of the imposed shear rate. From the stress-strain slope in this region (with $\sigma<\sigma_{\mathrm{y}}$ ), we extract a modulus $G \approx 11 \mathrm{~Pa}$ which, given the uncertainties involved (scattered data at short times), is consistent with the low-frequency plateau value $G_{\mathrm{p}}{ }^{\mathrm{r}}$ of the gel in Fig. 3. While at higher shear rates the system response is dominated by hydrodynamics, the contribution from the inherent system dynamics becomes non-negligible and progressively more important for $\dot{\gamma} \leq \dot{\gamma}_{\mathrm{c}}$. In Fig. 6(B), the stress data are normalized with the yield stress and plotted against strain. One can observe that at the highest strain rate, there is a broader transition from the peak to steady state. This may reflect reduced stress localization (as compared to lower rates), hence more gradual drop of stress to steady state, apparently due to shear-induced local cooperative rearrangements, and a tendency for more ductile-like yielding [Colombo et al. (2009)]. Figure 6(C) depicts the yield stress versus shear rate and shows that indeed, in this regime, $\sigma_{\mathrm{y}}$ is independent of the rate and has a plateau value $\sigma_{\mathrm{y}, \mathrm{p}} \approx 5 \mathrm{~Pa}$ which conforms to $\sigma_{\mathrm{y}, \mathrm{p}}=G_{\mathrm{p}}^{\mathrm{r}} \gamma_{\mathrm{y}}$, with $G_{\mathrm{p}}{ }^{\mathrm{r}}=10 \mathrm{~Pa}$ (Fig. 3). This low-rate behavior can be attributed to extremely localized (fracturelike) yield events and is suggestive of the particular microscopic yielding mechanism of the gel. According to Varnik and co-workers (2004), in short-range attractive glassy systems, when the system is sheared at rates below $\dot{\gamma}_{c}$, a yield stress is associated with enhanced stress release due to inherent structural relaxation (local intranetwork relaxation) and no longer (or else only weakly) depends on the strain rate.

\section{Gel characteristics and yielding process}

A remaining question pertains to the length scale involved in the depletion gel and its yielding process. From the linear viscoelastic data at $20^{\circ} \mathrm{C}$ (Fig. 3), we can extract the correlation length of density fluctuations, $\xi \approx\left(k_{\mathrm{B}} T / G_{\mathrm{P}}\right)^{1 / 3}=74 \mathrm{~nm}$. This average length is larger than the nominal diameter of a single shrunk star particle of about $29 \mathrm{~nm}$ 
(see Sec. III A), which confirms the existence of a percolating networklike structure. The latter should be expected for this range of star and linear polymer fractions [Trappe and Sandkühler (2004)]. Note that since there is no evidence for phase separation in the system and we have not studied other gel fractions, we refrain from further discussing this length scale in the context of arrested phase separation [Gibaud and Schurtenberger (2009); Conrad et al. (2010)]. Further, to obtain an "activation length" characterizing the yielding process, we fitted the yield stress curve in the inset of Fig. 6 with the Eyringtype cooperative model used originally to describe yielding during the deformation of linear polyethylene [Fotheringham and Cherry (1978)] and later applied to glassy systems [Varnik et al. (2004); Richeton et al. (2007)]

$$
\sigma_{\mathrm{y}}(\dot{\gamma})=\sigma_{\mathrm{y}, \mathrm{p}}+2 k_{\mathrm{B}} T / V^{*} \sinh \left[\left(\dot{\gamma} / \dot{\gamma}^{*}\right)^{1 / n}\right],
$$

where $V^{*}$ is the so-called activation volume, representing the volume of the subunit of material involved in the yielding process (i.e., the length scale of local rearrangements under shear), $\dot{\gamma} *$ is the characteristic thermally activated strain rate, and $n$ is a material parameter reflecting the cooperative motions of the star particles. The line the inset of Fig. 6(C) represents the nonlinear fit of Eq. (3) from which we extracted the activation length for cooperative rearrangements during yielding, $l^{*}=V^{* 1 / 3} \approx 77 \mathrm{~nm}$. The fitting also gave $n=0.7<1$, which indicates that the degree of cooperativity in the gel is low and that the single-yield event is highly localized. This means that at low rates, where the yield stress is independent of strain rate, no dissipative processes contribute to yielding but rather only local, elastic fracturelike events, which depend on strain only. Many such localized microyield events take place and eventually bring about macroscopic failure (yielding) of the gel. This may be thought of as a gradual procession of the yielding process, from the onset of liquidlike response, i.e., rupture of the weakest bonds, to complete fluidization [Hsiao et al. (2012); Studart et al. (2011); Furst and Pantina (2007)]. Note that a rate-independent yield stress has been reported in recent systematic studies of yielding in colloid-polymer depletion gels [Koumakis and Petekidis (2011); Koumakis et al. (2013)]. The (often observed) two-step yielding and microstructural rearrangement phenomena in gels are consistent with the present scenario [Koumakis and Petekidis (2011); Chan and Mohraz (2012)]. We note that the type of yielding discussed here for low density gels, i.e., a progression of localized bond-rupture events along mesoscopic percolating strands, was recently described by a bond-rupture model [Sprakel et al. (2011)].

The characteristic shear rate was found to be $\dot{\gamma} * \approx 0.032 \mathrm{~s}^{-1}$, which in fact represents the frequency of hopping attempts for yield elements of size $l^{*}$ involved in elementary shear motions within the star polymer gel. This characteristic rate is identified with the critical threshold value $\dot{\gamma}_{c}$ marking the onset of shear rate-dependent yield stress, in reasonable agreement with the experimental data [Fig. 6(C)]. Below this critical rate, the yielding behavior is dictated by the energy landscape of the gel and the localization of the fracturelike processes, whereas above it, the imposed shear modifies the energy barriers that the single activation volumes must overcome, and yielding is induced.

\section{Thermoreversible melting of the depletion star gel}

Since the osmotic pressure-which is at the origin of the depletion mechanismscales with kT, a depletion gel derived from hard colloid-polymer mixtures is not expected to melt upon heating. However, we have recently shown that gel melting can be also induced by heating [Truzzolillo et al. (2013b)], in addition to mechanical energy as already discussed. In particular, by performing dynamic frequency sweep measurements 
with a depletion star gel (with $\Phi_{\mathrm{L}}=2.24$ ) over a temperature range $20^{\circ} \mathrm{C}<T<90^{\circ} \mathrm{C}$, we observed a transition to liquidlike response between 60 and $80^{\circ} \mathrm{C}$, and eventually pure Newtonian response at $90^{\circ} \mathrm{C}$ [Fig. 7(A)]. This observation was also corroborated by temperature sweep data at fixed frequency $\left(\omega=10^{-2} \mathrm{rad} / \mathrm{s}\right)$. The melting temperature was estimated using the Winter-Chambon (1986) criterion to be $T_{\text {melt }}=\left.T\right|_{G^{\prime}=G^{\prime \prime}}=67.5 \pm 0.5^{\circ} \mathrm{C}$ [Truzzolillo et al. (2013b) $]$.

This result appears counterintuitive at first glance: A gel formed by a mixture of poly (methyl methacrylate) (PMMA) hard spheres and polystyrene chains [Pham et al. (2008)] at a fraction of 0.2 (similar to that of the shrunk star in the gel state) was indeed found to be invariant to heating, confirming the purely entropic origin of the depletion phenomenon [Truzzolillo et al. (2013b)]. Hence, an energetic contribution is at work in the starlinear polymer gel case. In fact, the unscreened van der Waals (vdW) interactions between the star cores, $U_{\mathrm{vdW}}$, were calculated from $R_{\text {core }}$. The largest possible value of $U_{\mathrm{vdW}}$ at the minimum star-star distance, when the cores touch each other, was found to be $U\left(2 R_{\min }\right)=0.025 k_{\mathrm{B}} T$, i.e., very small to explain gelation [Truzzolillo et al. (2013b)]. Since vdW interactions do not explain thermally induced melting of the star gel, we proposed a conformational change of the star arms on heating, akin to the well-known
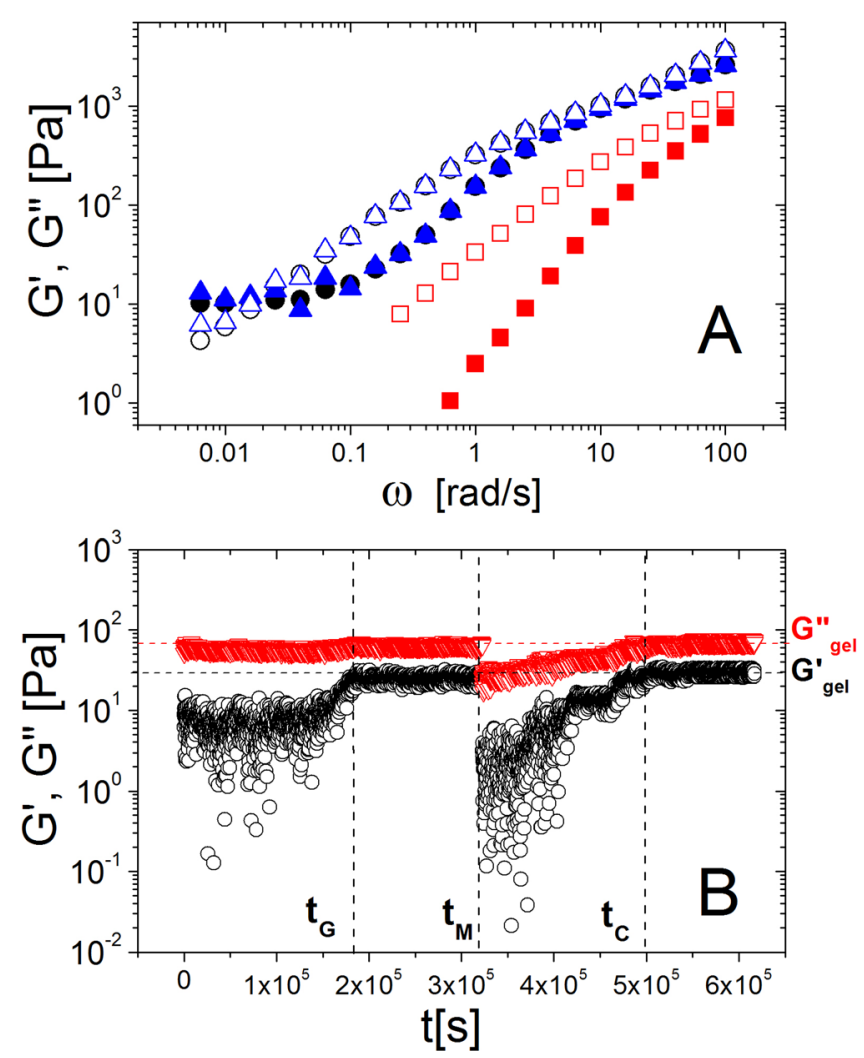

FIG. 7. (A) Linear viscoelastic spectra for mixtures $\mathrm{s} 362-2 / \mathrm{c} 6$ with $\Phi_{\mathrm{s}}=3.75$ and $\Phi_{\mathrm{L}}=2.24$ (gel state) during a thermal cycle. Black circles $20^{\circ} \mathrm{C}$ (initial state); red squares: $90^{\circ} \mathrm{C}$ (melting); blue triangles: $20^{\circ} \mathrm{C}$ (after melting). (B) Time evolution of the moduli $G^{\prime}$ (open black circles) and $G^{\prime \prime}$ (open red triangles) at fixed frequency for the gel at $20^{\circ} \mathrm{C}$ after rejuvenation. At time $t=t_{\mathrm{G}}=1.8 \times 10^{5} \mathrm{~s}$, gelation occurs spontaneously. At time $t=t_{\mathrm{M}}=3.2 \times 10^{5} \mathrm{~s}$, thermal melting occurs upon increasing the temperature $\left(20^{\circ} \mathrm{C} \longrightarrow 90^{\circ} \mathrm{C}\right)$. After probing linear viscoelasticity (panel $\mathrm{A}$ ), the temperature was set again to $20^{\circ} \mathrm{C}$. The gel coarsening time was $t_{\mathrm{C}}-t_{\mathrm{M}}=1.8 \times 10^{5} \mathrm{~s}$. 
dry-to-wet transition in polymeric brushes [Gast and Leibler (1986); Lindenblatt et al. (2001); Chevigny et al. (2010); Srivastava et al. (2012)]. It is important to note that the currently observed effect, in a nearly athermal solvent, is very different from that reported in intermediate-quality solvents (polybutadiene stars in decane or tetradecane), where heating improved the solvent quality and led to substantial swelling, and hence to a liquid-to-glass transition [Likos et al. (1998b); Kapnistos et al. (2000)]. In the current system, we rationalized this conformational change by the chemically different nature of the Si-containing core and the polybutadiene arms of the stars, with different thermal expansion coefficients: $\kappa_{\text {core }} \approx 10^{-4} \mathrm{~K}^{-1}$ [Mazo et al. (1999)], $\kappa_{\mathrm{PBD}}=6.6 \cdot 10^{-4} \mathrm{~K}^{-1}$. The internal strain generated by this mismatch may be non-negligible and likewise its influence on the macroscopic properties of the materials [Pigozzi et al. (2009); Astefanoaei et al. (2011)]. We estimated this strain from the increase in temperature $\Delta T$ and the thermal expansion mismatch $\Delta \kappa=\kappa_{\mathrm{PBD}}-\kappa_{\mathrm{Si}}$, as $\Delta \varepsilon(\Delta T)=\Delta \kappa \Delta T$. Therefore, the respective thermally induced radial deformation is $\Delta l=R_{\text {core }} \Delta \varepsilon$.

The gel melting observed upon heating is thermoreversible: When the liquid at $90{ }^{\circ} \mathrm{C}$ is cooled back to $20^{\circ} \mathrm{C}$, the exact original gel is recovered, as demonstrated by the identical dynamic frequency sweep data of Fig. 7(A). We now examine the kinetics of the gel-liquid-gel transition by means of dynamic time sweep measurements in the linear viscoelastic regime. As seen in Fig. 7(B), following preshear rejuvenation according to the protocol discussed in Secs. IIB and III A, the depletion gel with $\Phi_{\mathrm{s}}=3.75$ and $\Phi_{\mathrm{L}}=2.24$ is formed and reaches a quasisteady state after a gelation time $t_{\mathrm{G}}$ of typically about $1.8 \times 10^{5} \mathrm{~s}$. Then, at time $t_{\mathrm{M}} \approx 3.2 \times 10^{5} \mathrm{~s}$, the gel is heated to $90^{\circ} \mathrm{C}$ and is transformed into a liquid [see also Fig. 7(A)]. Subsequent cooling to the original temperature of $20^{\circ} \mathrm{C}$ leads to reformation of the gel with exactly the same moduli. Interestingly, the kinetics of gel reformation are on the order of the aging kinetics, i.e., the time $t_{\mathrm{C}}$ for "quiescent" coarsening of the gel is about $1.8 \times 10^{5} \mathrm{~s}$. Of course this time exceeds the time required for temperature equilibration (about $60 \mathrm{~s}$ ). This experimental evidence suggests that mechanical and thermal rejuvenations of the gel are equivalent. The different steady states involved in this thermoreversible gelation are illustrated in Fig. 8. The original depletion gel is rejuvenated under shear [Fig. 8(A)], and upon shear cessation, it reforms after a long coarsening time [Figs. 7(B) and 8(B)]. Subsequent heating changes the conformation of the stars and leads to a liquid [Fig. 8(C)]. Finally, cooling to the initial temperature results in reformation of the original gel with the same coarsening time [Figs. 7(B) and 8(D)].

\section{Sketching a state diagram}

We summarize the main findings from this work in the form of a state diagram, depicted in Fig. 9. It represents the different states of the star-linear polymer mixtures studied for different effective volume fractions of star $\Phi_{\mathrm{s}}$ and added linear chains $\Phi_{\mathrm{L}}$. The line at $\Phi_{\mathrm{s}}=3.75$ corresponds to the systems discussed in the present work. We added data for the line $\Phi_{\mathrm{s}}=2$ taken from our earlier work [Truzzolillo et al. (2013a)], and in order to determine the extension of the gel region, we performed additional SAOS measurements from $\Phi_{\mathrm{s}}=2.0$ to $\Phi_{\mathrm{s}}=3.75$ for selected mixtures in the absence of solvent. The liquid-to-gel transition was observed for $3.0 \leq \Phi_{\mathrm{s}} \leq 3.2$ : For $\Phi_{\mathrm{s}}=3.2$, the onset of a plateau modulus $\left(G^{\prime} \sim \omega^{0}\right)$ at low frequencies was unambiguously observed. An extensive rheological study of this solvent-free region is currently underway and will be presented in a future publication. One can observe that for $\Phi_{\mathrm{s}}>1$, the star solution is glassy. Adding small amounts of linear polymers does not change the state of the system, even though the glass becomes softer [Fig. 2(A)]. Further addition of linear chains results in a 


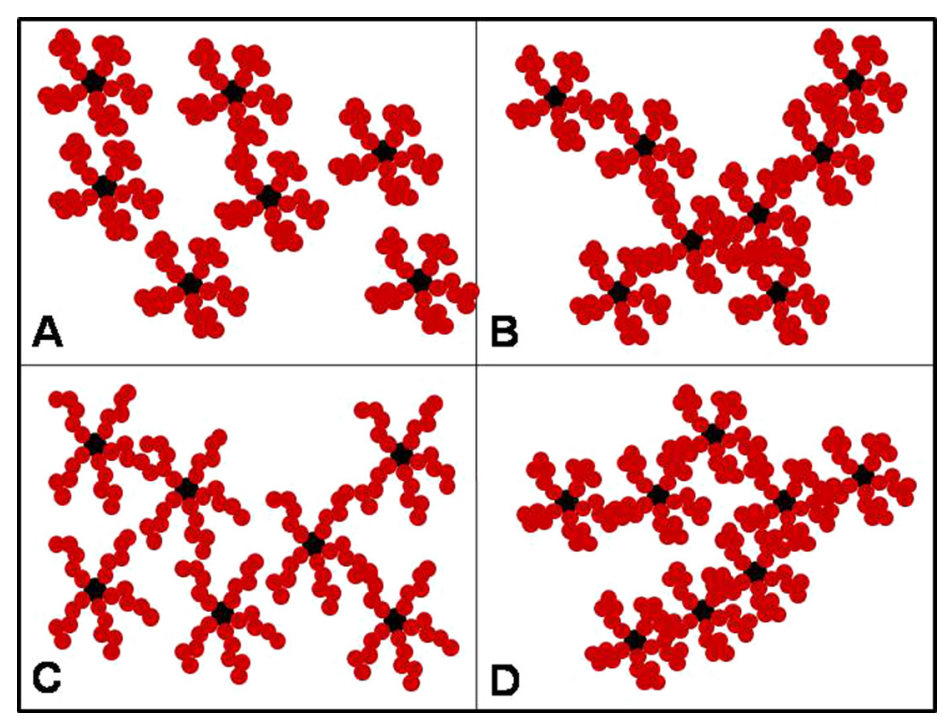

FIG. 8. The four cartoons show the star polymer gel after preshear for different waiting times $(t): t=0$ : sample is rejuvenated, i.e., liquidlike (A); $t_{\mathrm{G}}<t<t_{\mathrm{M}}$ : Sample ages and the gel is formed; the shrunk stars are attracted via depletion and form a percolated network (B); $t_{\mathrm{M}}<t<t_{\mathrm{C}}$ : The gel melts upon heating and a liquidlike sample results, but the star size is larger than in (A) due to small thermal swelling and change of conformation, as discussed in the text $(\mathrm{C}) ; t>t_{\mathrm{C}}$ : Upon cooling, with time the percolated gel network is reformed (D).

higher osmotic pressure and hence melting of the star glass. Note that the larger $\Phi_{\mathrm{s}}$ is, the larger is the osmotic pressure needed to break the cages, which is reasonable given the fact that the higher- $\Phi_{\mathrm{s}}$ glasses are stronger. Further addition of linear chains leads to a reentrant depletion star gel. Note that such gels have been probed for different original star glasses in the range $3.2 \leq \Phi_{\mathrm{s}} \leq 3.75$, whereas for $\Phi_{\mathrm{s}}<3$, no reentrant gel is obtained.

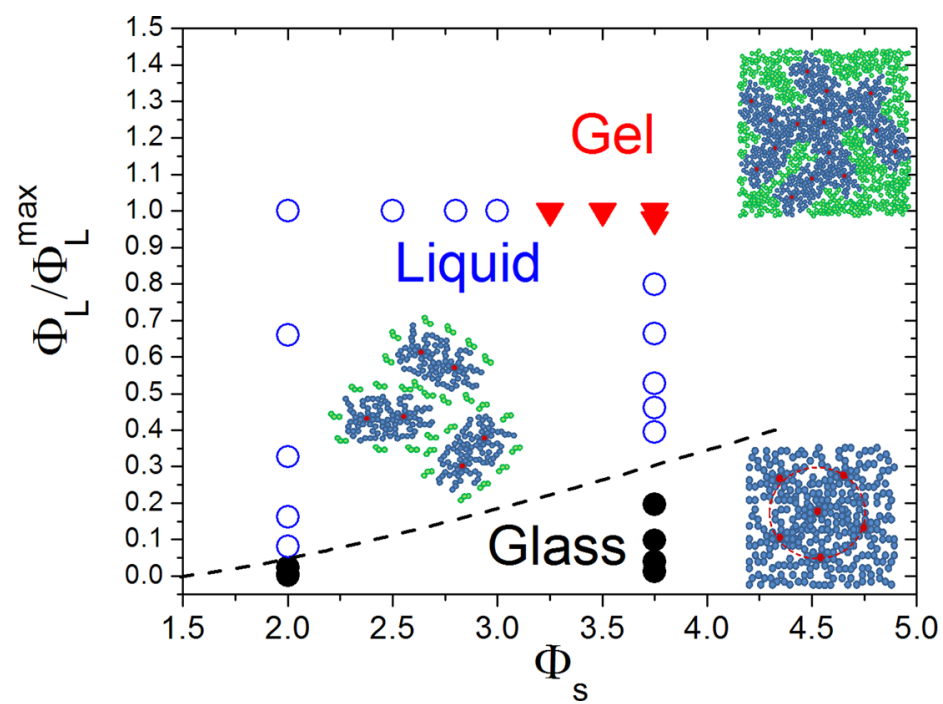

FIG. 9. State diagram for mixtures s362-2/c6. Repulsive glass (black full circles), liquid (empty blue circles), gel (red full circles). The linear polymer concentration has been normalized with the maximum concentration of chains corresponding to the absence of solvent $\left(\Phi_{\mathrm{L}} / \Phi_{\mathrm{L}}{ }^{\mathrm{max}}=0\right.$ : No linear chains; $\Phi_{\mathrm{L}} / \Phi_{\mathrm{L}}{ }^{\mathrm{max}}=1$ : No solvent $)$. The line separating the glass from the liquid regime is drawn to guide the eye. 
This is qualitatively consistent with the generic trend in colloid-polymer mixtures [Pham et al. (2002); Eberle et al. (2011)], although the diagram of Fig. 9 is restricted to the glassy star regime.

\section{GENERIC GUIDELINES FOR COLLOID-POLYMER MIXTURES}

Given the behavior of soft spheres presented in this work, a challenging question concerns what generic changes variations in softness can impart on the state of colloidpolymer mixtures. We attempted to address this point with Fig. 10, where we plotted the apparent plateau modulus of the solid (normalized to that of the original repulsive glass) as function of the effective volume fraction of the added nonadsorbing linear polymer. Note that we cannot compare the systems at the same particle volume fractions, as for the star case (and any soft colloidal particle), this quantity is ill-defined [Royall et al. (2013)]; however, we use an apparent value (discussed in Sec. II A) which is above 1 for stars (and other soft particles), well within the glass regime. The hard-sphere colloidpolymer case is depicted in Fig. 10(A) with data drawn from the literature [Pham et al. (2008)]. One observes fast, substantial softening of the original repulsive glass. With further increase in the linear polymer fraction, the glass melts and a small ergodic pocket represents the liquid. In the case of the cited Pham et al. (2008) work, this pocket was suppressed due to the high polydispersity of the particles, but in general, it is expected as

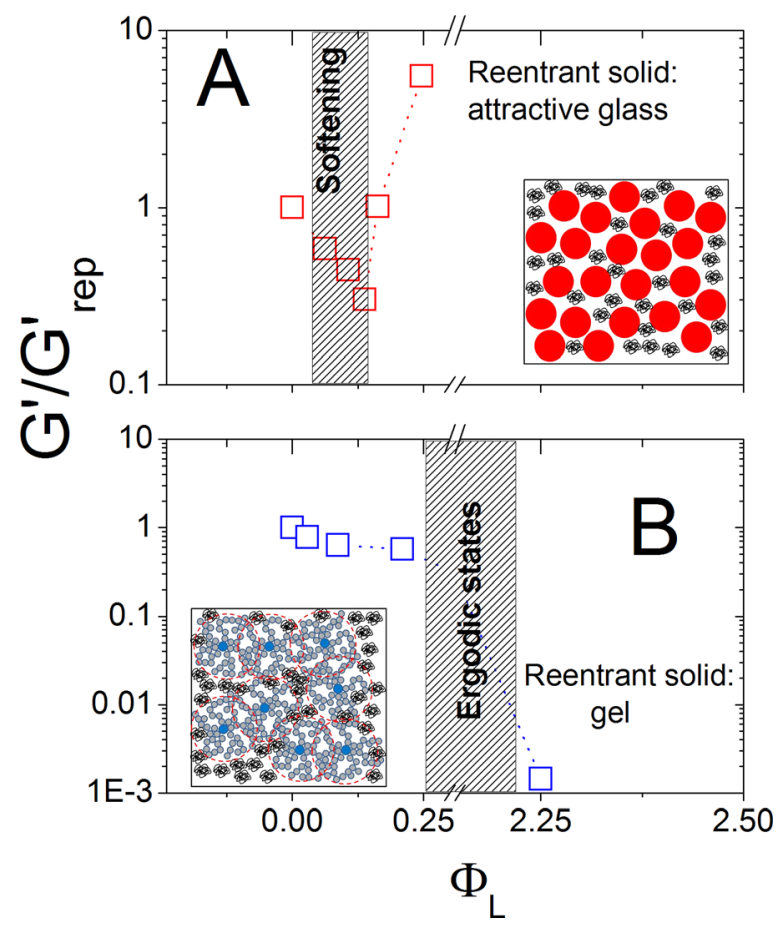

FIG. 10. Storage moduli $G^{\prime}$ for HS-polymer mixtures (A) and star-linear polymer mixtures (B) at $\omega=10^{-2} \mathrm{rad} / \mathrm{s}$ as function of the linear polymer concentration. The moduli are normalized to the modulus of the repulsive glass $G_{\text {rep }}$ in absence of chains. (A) Data are taken from Pham et al. (2008). The system is composed by PMMA spheres dispersed in cis-decalin with a hydrodynamic radius $R_{\mathrm{h}}=130 \mathrm{~nm}$ at a volume fraction $\Phi_{\mathrm{HS}}=0.60$, and polystyrene linear chains with a molar mass $M_{\mathrm{w}}=143400 \mathrm{~g} / \mathrm{mol}$ and a radius of gyration $R_{\mathrm{g}}=11 \mathrm{~nm}$. (B) Star-linear polymer solutions at $\Phi_{\mathrm{s}}=3.75$ as described in Sec. II. The insets show sketches for the two systems. 
discussed above and in Pham et al. (2002); hence this is qualitatively similar to the present soft colloidal mixtures. Eventually a reentrant solid is formed, identified with the attractive glass, having a plateau modulus much higher than the repulsive glass. It is worth pointing out that the reentrance phenomenon is more general and applies to spherical colloids interacting via different forces, as well as nonspherical particles [Kramb et al. (2011)].

On the other hand, the soft-sphere star colloid-polymer mixture exhibits some qualitative differences, as seen in Fig. 10(B): Softening of the original repulsive glass upon adding the linear polymer is weak. Subsequently, a large liquid pocket is formed. We emphasize the relatively large extent of this pocket, in contrast to the hard-sphere analogues [Pham et al. (2002)]. We attribute the broad range of linear polymer fractions leading to the liquid state in the present case to the fact that the star polymers are osmotically shrunk; hence, their effective volume fraction is reduced. Note that the linear polymer fraction range where the ergodic pocket appears depends on parameters like the (glassy) star fraction and the size ratio, and hence, it is not compared quantitatively with the hard sphere mixtures. Finally, due to this effect, the reentrant solid is a gel, with a much lower modulus as compared to the original star glass (almost three decades). Hence, the qualitative and quantitative differences are mainly due to the ability of stars to shrink and change their conformation.

The above comparison is by no means complete, but it constitutes a first step in trying to put together the properties of model hard and soft colloidal systems. It may be suggestive of pathways to follow to address questions related to the processing and/or performance of soft composites.

\section{CONCLUDING REMARKS}

Using the star polymer paradigm, we have shown that repulsive soft colloidal particles exhibit a variety of tunable metastable states due to their flexible shape, which can adjust in response to external stimuli. Adding linear homopolymers to a repulsive star glass exerts osmotic forces which lead to star shrinkage and depletion. The result is gradual weakening of the original glass and its melting. Eventually, further addition of linear polymers leads to a unique reentrant gel state due to fact that the shrunk stars attract each other entropically and form a percolated network. This gel is characterized by a very low modulus, very long coarsening time upon rejuvenation, and a shear-induced yielding process dominated by a procession of localized fracturelike events. From analysis of the linear viscoelasticity and the shear rate-dependent yield stress, the typical length scale of the gel involved in the yielding process, which is its correlation length, is much larger than the diameter of the shrunk stars. We further find that this depletion gel can melt thermoreversibly, due to the star's hybrid core-shell microstructure, which can explain the changes in conformation of the arms with variations in temperature. Interestingly, mechanical and thermal annealing of the gel after its melting (rejuvenation) is equivalent, as they have the same coarsening kinetics and lead to identical steady-state moduli.

The reentrant gels derived from glassy colloid-polymer mixtures are unique to soft deformable particles. This sets these systems apart from hard colloid-polymer mixtures and could be potentially useful in handling soft composites with low moduli. Hence, the direct comparison of hard-sphere and soft-sphere composites may affect applications of colloidal mixtures in the food, pharmaceutical, and chemical industries [e.g., Mezzenga and Fischer (2013)]. In addition, the intriguing tunability of soft colloids may provide new insights into the physics of metastable states of soft matter. 


\section{ACKNOWLEDGMENTS}

The authors thank Antje Larsen for assistance with the DLS characterization and Emanuela Del Gado and Jader Colombo for enlightening discussions. Financial support from the EU (ITN-COMPLOIDS FP7-234810, FP7 Infrastructure ESMI, GA 262348 and FP7-SMALL-Nanodirect CP-FP-213948) and the Natural Science and Engineering Research Council of Canada (NSERC) is gratefully acknowledged.

\section{References}

Asakura, S., and F. Oosawa, "Interaction between particles suspended in solutions of macromolecules," J. Polym. Sci. 33, 183-192 (1958).

Astefanoaei, I., I. Dumitru, and A. Stancu, "Induced thermal stresses in core-shell magnetic particles," IEEE Trans. Magn. 47(10), 3829-3832 (2011).

Bonnecaze, R. T., and M. Cloitre, "Micromechanics of soft particle glasses," Adv. Polym. Sci. 236, 117-161 (2010).

Camargo, M., and C. N. Likos, "Unusual features of depletion interactions in soft polymer-based colloids mixed with linear homopolymers,” Phys. Rev. Lett. 104, 078301 (2010).

Cates, M. E., M. Fuchs, K. Kroy, W. C. K. Poon, and A. M. Puertas, "Theory and simulation of gelation, arrest and yielding in attracting colloids,” J. Phys.: Condens. Matter 16, S4861-S4875 (2004).

Chan H. K., and A. Mohraz, "Two-step yielding and directional strain-induced strengthening in dilute colloidal gels,” Phys. Rev. E 85, 041403 (2012).

Chevigny, C., J. Jestin, D. Gigmes, R. Schweins, E. Di-Cola, F. Dalmas, D. Bertin, and F. Boué, "Wet-to-Dry conformational transition of polymer layers grafted to nanoparticles in nanocomposite," Macromolecules 43, 4833-4837 (2010).

Colombo, J., A. Widmer-Cooper, and E. Del Gado, "Microscopic picture of cooperative processes in restructuring gel networks," Phys. Rev. Lett. 110, 198301 (2013).

Conrad, J. C., H. M. Wyss, V. Trappe, S. Manley, K. Miyazaki, L. J. Kaufman, A. B. Schoefield, D. R. Reichman, and D. A. Weitz, "Arrested fluid-fluid phase separation in depletion systems: Implications of the characteristic length on gel formation and rheology," J. Rheol. 54, 421-438 (2010).

Daoud, M., and J. P. Cotton, "Star shaped polymers: A model for the conformation and its concentration dependence," J. Phys. 43, 531-538 (1982).

Datta, S., D. D. Gerrard, T. S. Rhodes, T. G. Mason, and D. A. Weitz, "Rheology of attractive emulsions," Phys. Rev. E. 84, 041404 (2011).

Del Gado, E., and W. Kob, "Length-scale dependent relaxation in colloidal gels," Phys. Rev. Lett. 98, 028303 (2007).

Eberle, A. P. R., N. J. Wagner, and R. Castaneda-Priego, "Dynamical arrest transition in nanoparticle dispersions with short-range interactions," Phys. Rev. Lett. 106, 105704 (2011).

Eckert, T., and E. Bartsch, "Reentrant glass transition in a colloid-polymer mixture with depletion attractions," Phys. Rev. Lett. 89, 125701-125704 (2002).

Eckert, T., and E. Bartsch, "The effect of free polymer on interactions and glass transition dynamics of microgel," Faraday Discussions 123, 51-64 (2003).

Erwin, B. M., D. Vlassopoulos, and M. Cloitre, "Rheological fingerprinting of an aging colloidal glass," J. Rheol. 54, 915-939 (2010).

Erwin, B. M., D. Vlassopoulos, M. Gauthier, and M. Cloitre, "Unique slow dynamics and aging phenomena in soft glassy suspensions of multiarm star polymers," Phys. Rev. E 83, 061402 (2011).

Fotheringham, D., and B. W. Cherry, "The role of recovery forces in the deformation of linear polyethylene," J. Mater. Sci. Lett. 13, 951-961 (1978).

Furst, E. M., and J. P. Pantina, "Yielding in colloidal gels due to nonlinear microstructure bending mechanics," Phys. Rev. E 75, 050402 (2007).

Gast, A. P., and L. Leibler, "Interactions of sterically stabilized particles suspended in a polymer solution," Macromolecules 19, 686-691 (1986). 
Gauthier, M., and A. Munam, "Synthesis of 1,4 polybutadiene dendrimer arborescent polymer hybrids," Macromolecules 43, 3672-3681 (2010).

Gibaud, T., and P. Schurtenberger, "A closer look at the arrested spinodal decomposition in protein solutions," J. Phys.: Condens. Matter 21, 322201 (2009).

Hoy, R. S., and G. S. Grest, "Entanglements of an end-grafted polymer brush in a polymeric matrix," Macromolecules 40, 8389-8395 (2007).

Hsiao, L. C., R. S. Newman, S. C. Glotzer, and M. J. Solomon, "Role of isostaticity and load-bearing microstructure in the elasticity of yielded colloidal gels," Proc. Natl. Acad. Sci. U.S.A. 109, 16029-16034 (2012).

Kamble, S., A. Pandey, S. Rastogi, and A. Lele, "Ascertaining universal feature of yielding of soft materials," Rheol. Acta 52, 859-865 (2013).

Kapnistos, M., D. Vlassopoulos, G. Fytas, K. Mortensen, G. Fleischer, and J. Roovers, "Reversible thermal gelation in soft spheres," Phys. Rev. Lett. 85, 4072-4075 (2000).

Koumakis, N., and G. Petekidis, "Two-step yielding in attractive colloids: Transition from gels to attractive glasses," Soft Matter 7, 2456-2470 (2011).

Koumakis, N., A. Pamvouxoglou, A. S. Poulos, and G. Petekidis, "Direct comparison of the rheology of model hard and soft particle glasses," Soft Matter 8, 4271-4284 (2012).

Koumakis, N., P. Ballesta, R. Besseling, W. C. K. Poon, J. F. Brady, and G. Petekidis, "Colloidal gels under shear: Strain-rate effects," AIP Conf. Proc. 1518, 365-371 (2013).

Kramb, R. C., and C. F. Zukoski, "Nonlinear rheology and yielding in dense suspensions of hard anisotropic colloids,” J. Rheol. 55, 1069-1084 (2011).

Kramb, R. C., R. Zhang, K. S. Schweizer, and C. F. Zukoski, "Re-entrant kinetic arrest and elasticity of concentrated suspensions of spherical and nonspherical repulsive and attractive colloids," J. Chem. Phys. 134, 014503 (2011).

Lekkerkerker, H. N. W., and R. Tuinier, Colloids and the Depletion Interaction, Lecture Notes in Physics, Vol. 833 (Springer, NY, 2011).

Likos, C. N., H. Löwen, A. Poppe, L. Willner, J. Roovers, B. Cubitt, and D. Richter, "Ordering phenomena of star polymer solutions approaching the $\Theta$ state,” Phys. Rev. E 58, 6299-6307 (1998b).

Likos, C. N., H. Löwen, M. Watzlawek, B. Abbas, O. Jucknischke, J. Allgaier, and D. Richter, "Star polymers viewed as ultrasoft colloidal particles," Phys. Rev. Lett. 80, 4450-4453 (1998a).

Lindenblatt, G., W. Schärtl, T. Pakula, and M. Schmidt, "Structure and dynamics of hairy spherical colloids in a matrix of nonentangled linear chains," Macromolecules 34, 1730-1736 (2001).

Mao, Y., M. E. Cates, and H. N. W. Lekkerkerker, "Depletion force in colloidal systems," Physica A 222, 10-24 (1995).

Masschaele, K., and J. Vermant, "Direct visualization of yielding in model two-dimensional colloidal gels subjected to shear flow," J. Rheol. 53, 1437-1460 (2009).

Matsen, M. W., and J. M. Gardiner, "Autophobic dewetting of homopolymer on a brush and entropic attraction between opposing brushes in a homopolymer matrix,” J. Chem. Phys. 115, 2794-2804 (2001).

Mazo, M. A., P. A. Zhilin, E. B. Gusarova, S. S. Sheiko, and N. K. Balabaev, "Computer simulation of intramolecular mobility of dendrimers," J. Mol. Liq. 82, 105-116 (1999).

Mewis, J., and N. J. Wagner, Colloidal Suspension Rheology (Cambridge University Press, NY, 2012).

Mezzenga, R., and P. Fischer, "The self-assembly, aggregation and phase transitions of food protein systems in one, two and three dimensions,” Rep. Prog. Phys. 76, 046601 (2013).

Moll, J., S. K. Kumar, F. Snijkers, D. Vlassopoulos, A. Rungta, B. C. Benicewicz, E. Gomez, J. Ilavsky, and R. H. Colby, "Dispersing grafted nanoparticle assemblies into polymer melts through flow fields," ACS Macro Lett. 2, 1051-1055 (2013).

Osman, M. A., A. Attalah, T. Schweizer, and H. C. Öttinger, "Particle-particle and particle-matrix interactions in calcite filled high-density polyethylene-Steady shear," J. Rheol. 48, 1167-1184 (2004).

Pham, K. N., A. M. Puertas, J. Bergenholtz, S. U. Egelhaaf, A. Moussaiud, P. N. Pusey, A. B. Schofield, M. E. Cates, M. Fuchs, and W. C. K. Poon, "Multiple glassy states in a simple model system," Science 296, 104-106 (2002).

Pham, K. N., G. Petekidis, D. Vlassopoulos, S. U. Egelhaaf, W. C. K. Poon, and P. N. Pusey, "Yielding behavior of repulsion- and attraction-dominated colloidal glasses," J. Rheol. 52, 649-676 (2008). 
Pigozzi, G., D. Mukherji, R. Gilles, P. Jencus, and C. Siemers, “The measurement of internal strain in core-shell $\mathrm{Ni}_{3} \mathrm{Si}(\mathrm{Al})-\mathrm{SiOx}$ nanoparticles,” Nanotechnology 20, 245704 (2009).

Poon, W. C. K., “The physics of a colloid-polymer mixture,” J. Phys.: Condens. Matter 14, R859-R880 (2002).

Puertas, A. M., M. Fuchs, and M. E. Cates, "Dynamical heterogeneities close to a colloidal gel," J. Chem. Phys. 121, 2813 (2004).

Putz, A. M. V., and T. I. Burghelea, "The solid-fluid transition in a yield stress shear thinning physical gel," Rheol. Acta 48, 673-689 (2009).

Ramakrishan, S., M. Fuchs, K. S. Schweizer, and C. F. Zukoski, "Entropy driven phase transitions in colloidpolymer suspensions: Tests of depletion theories," J. Chem. Phys. 116, 2201 (2002).

Ramos, L., and L. Cipelletti, "Intrinsic aging and effective viscosity in the slow dynamics of a soft glass with tunable elasticity," Phys. Rev. Lett. 94, 158301 (2005).

Raphael, E., P. Pincus, and G. H. Fredrickson, "Conformation of star polymers in high-molecular weight solvents," Macromolecules 26, 1996-2006 (1993).

Reiter, G., and R. Khanna, "Negative excess interfacial entropy between free and end-grafted chemically identical polymers," Phys. Rev. Lett. 85, 5599-5602 (2000).

Richeton, J., S. Ahzi, and L. Daridon, "Thermodynamic investigation of yield-stress models for amorphous polymers," Philos. Mag. 87, 3629-3643 (2007).

Rogers, S. A., B. M. Erwin, D. Vlassopoulos, and M. Cloitre, "A sequence of physical processes determined and quantified in LAOS: Application to a yield-stress fluid," J. Rheol. 55, 435-458 (2011).

Roovers, J., and J. Ding, "Carbosilane dendrimers," in Silicon-Containing Dendritic Polymers, Advances in Silicon Science, Vol. 2 (Springer, New York, 2009), pp. 31-74.

Rouyer, F., S. Cohen-Addad, M. Vignes-Adler, and R. Höhler, "Dynamics of yielding observed in a threedimensional aqueous foam,” Phys. Rev. E. 67, 021405 (2003).

Royall, C. P., W. C. K. Poon, and E. R. Weeks, "In search of colloidal hard spheres," Soft Matter 9, 17-27 (2013).

Rueb, C. J., and C. F. Zukoski, "Viscoelastic properties of colloidal gels,” J. Rheol. 41, 197-218 (1997).

Russel, W. B., D. A. Saville, and W. R. Schowalter, Colloidal Dispersions (Cambridge University Press, NY, 1989).

Shah, S. A., Y.-L. Chen, K. S. Schweizer, and C. F. Zukoski, "Viscoelasticity and rheology of depletion flocculated gels and fluids," J. Chem. Phys. 119, 8747 (2003).

Shih, W. H., W. Y. Shih, S. I. Kim, J. Liu, and I. A. Aksay, "Scaling behavior of the elastic properties of colloidal gels," Phys. Rev. A 42, 4772-4779 (1990).

Sprakel, J., S. B. Lindström, T. E. Kodger, and D. A. Weitz, "Stress enhancement in the delayed yielding of colloidal gels,” Phys. Rev. Lett. 106, 248303 (2011).

Srivastava, S., P. Agarwal, and L. A. Archer, "Tethered nanoparticle-polymer composites: Phase stability and curvature," Langmuir 28, 6276-6281 (2012).

Stiakakis, E., D. Vlassopoulos, C. N. Likos, J. Roovers, and G. Meier, "Polymer-mediated melting in ultrasoft colloidal gels," Phys. Rev. Lett. 89, 208302 (2002).

Stiakakis, E., G. Petekidis, D. Vlassopoulos, C. N. Likos, H. Iatrou, N. Hadjichristidis, and J. Roovers, "Depletion and cluster formation in soft colloid-polymer mixtures," Europhys. Lett. 72, 664-670 (2005).

Studart, A. R., E. Amstadb, and L. J. Gaucklerb, "Yielding of weakly attractive nanoparticle networks," Soft Matter 7, 6408-6412 (2011).

Trappe, V., and P. Sandkühler, "Colloidal gels_-Low-density disordered solid-like states," Curr. Opin. Colloid Interface Sci. 8, 494-500 (2004).

Truzzolillo, D., D. Vlassopoulos, and M. Gauthier, "Osmotic interactions, rheology and arrested phase separation in star-linear polymer mixtures," Macromolecules 44, 5043-5052 (2011).

Truzzolillo, D., D. Vlassopoulos, and M. Gauthier, "Rheological detection of caging and solid-liquid transitions in soft colloid-Polymer mixtures,” J. Non-Newtonian Fluid Mech. 193, 11-20 (2013a).

Truzzolillo, D., D. Vlassopoulos, M. Gauthier, and A. Munam, "Thermal melting in depletion gels of hairy nanoparticles," Soft Matter 9, 9088-9093 (2013b).

Tuinier, R., J. K. G. Dhont, and C. G. de Kruif, "Depletion-induced phase separation of aggregated whey protein colloids by an exocellular polysaccharide,” Langmuir 16, 1497-1507 (2000). 
Varadan, P., and M. J. Solomon, "Shear-induced microstructural evolution of a thermoreversible colloidal gel," Langmuir 17, 2918-2919 (2001).

Varnik, F., L. Bocquet, and J. L. Barrat, “A study of the yield stress in a binary Lennard-Jones glass,” J. Chem. Phys. 120, 2788 (2004).

Vincent, B., J. Edwards, S. Emmett, and A. Jones, "Depletion flocculation in dispersions of sterically-stabilized particles ('soft spheres')," Colloids Surf. 18, 261-281 (1986).

Vlassopoulos, D., and G. Fytas, "From polymers to colloids: Engineering the dynamic properties of hairy particles,” Adv. Polym. Sci. 236, 1-54 (2010).

Vlassopoulos, D., G. Fytas, S. Pispas, and N. Hadjichristidis, "Spherical polymeric brushes viewed as soft colloidal particles: Zero-shear viscosity," Physica B 296, 184-189 (2001).

Vrij, A., "Polymers at interfaces and the interaction in colloidal dispersions," Pure Appl. Chem. 48, 471-483 (1976).

Wiemann, M., N. Willenbacker, and E. Bartsch, "Effects of cross-link density on re-entrant melting of microgel colloids," Colloids Surf., A 413, 78-83 (2012).

Wilk, A., S. Huißmann, E. Stiakakis, J. Kohlbrecher, D. Vlassopoulos, C. N. Likos, G. Meier, J. K. G. Dhont, G. Petekidis, and R. Vavrin, "Osmotic shrinkage in star-linear polymer mixtures,” Eur. Phys. J. E. 32, 127-134 (2010).

Winter, H. H., and F. Chambon, "Analysis of linear viscoelasticity of a crosslinking polymer at the gel point," J. Rheol. 30, 367-382 (1986).

Zaccarelli, E., "Colloidal gels: Equilibrium and non-equilibrium routes," J. Phys: Condens. Matter 19, 323101-323151 (2007).

Zaccone, A., H. Wu, and E. Del Gado, "Elasticity of arrested short-ranged attractive colloids: Homogeneous and heterogeneous glasses," Phys. Rev. Lett. 103, 208301 (2009).

Zhou, L.-L., and J. Roovers, "Synthesis of novel carbosilane dendritic macromolecules," Macromolecules 26, 963-968 (1993). 